\title{
Statistical Analysis for Long Term Correlations in the Stress Time Series of Jerky Flow
}

\author{
D. Kugiumtzis ${ }^{1}$ and E. C. Aifantis ${ }^{2}$ \\ 'Department of Mathematical, Physical and Computational Sciences, Faculty of Engineering, \\ Aristotle University of Thessaloniki, GR-54006, Thessaloniki, Greece \\ ${ }^{2}$ Labaratory of Mechanics and Materials, Faculty of Engineering, Aristotle University of \\ Thessaloniki, GR-54006, Thessaloniki, Greece
}

\begin{abstract}
Stress time series from the PLC effect typically exhibit stick-slips regarding the upload and download of total stress. These data contain strong short-term correlations of a nonlinear type. We investigate whether there are also long term correlations, i.e. the successive up-down patterns are generated by a deterministic mechanism. A statistical test is conducted for the null hypothesis that the sequence of the up-down patterns is totally random. The test is constructed by means of surrogate data, suitably generated to represent the null hypothesis. Linear and nonlinear estimates are used as test statistics, namely autocorrelation, mutual information and Lyapunov exponents, which are found to have proper performance for the test. The test is then applied to three stress time series under different experimental conditions. Rejections could be obtained for one of them and not with all statistics. From the overall results we cannot conclude that the underlying mechanism to the PLC effect has long memory.
\end{abstract}

Key word index: time series, nonlinearity, surrogate data test, PLC effect

\section{INTRODUCTION}

The Portevin-Le Chatelier (PLC) effect or jerky flow is one of the best studied forms of plastic instability in many metallic alloys when tensile specimens are deformed in a certain range of strain rates and temperatures. A distinct characteristic of PLC effect is the up-load and down-load of the total stress due to atomic dislocations $/ 1 /$. As a result the stress time series is comprised of successive stick-slip patterns, i.e. slow rather linear up-trends followed by fast down-trends. First principle models, suggested in the literature, 
reproduce partially this feature $/ 2,3,4,5,6 /$. Recently, data analysis using nonlinear methods gives evidence for nonlinear and chaotic behavior $/ 7,8,9 /$.

Nonlinear methods, mostly based on chaos theory, have been applied to real data from different fields with varying success $110,11 \%$. These methods provide estimates of dynamical characteristics of the underlying system, such as topological or fractal dimension, entropy and Lyapunov exponents, as well as sophisticated data driven modes. However, the estimates are meaningful if there is evidence that the underlying system is indeed nonlinear deterministic and eventually chaotic. Regarding the nonlinearity in the data, an indirect approach, namely testing the null hypothesis that the data are linear stochastic, has gained much interest in recent years. The test employs surrogate data to form the empirical distribution of the test statistic under the null hypothesis $/ 12,13,14 /$.

The methodology of nonlinear dynamics, including the surrogate data test for nonlinearity, has been applied recently to data from the PLC effect. In a number of experiments of occurrence of the PLC effect, nonlinear techniques were used aiming at characterising the structure of the stress times series. Within a certain experimental range, jerky flow was reported to exhibit chaotic behaviour for single crystals of $\mathrm{Cu}-\mathrm{Al}$ alloys /8, 5/ as well as for Al-Mg polycrystals /9, 7/. Moreover, for both crystals, the surrogate data test gave evidence for the existence of nonlinear dynamics. However, one could argue that this evidence is solely due to the presence of strong deterministic structure at small time scales within the upload or download phase, a quite obvious form of nonlinear dynamics.

In this work, we direct the statistical analysis to a different time scale. We focus on correlations at a larger time scale that spans over the stick-slips, that is we investigate whether there is long term deterministic structure in addition to the short term nonlinear dynamics that forms the stick-slip patterns. The data analysis is done by means of hypothesis testing, where the null hypothesis is that the stick- slip sequence is random. For this we introduce an algorithm generating surrogate data with the same stick-slip patterns at a random order and we apply several linear and nonlinear statistics. We use three stress time series from single crystals at different experimental conditions.

The outline of the paper is as follows. In Section 2, the stress data are described and in Section 3, the results of the standard nonlinear analysis on these are reviewed. In Section 4, the surrogate test for the hypothesis of random stick-slips is described, and in Section 5, the results of the application of the test to the stress data are presented. Finally, a discussion follows in Section 6.

\section{THE STRESS DATA}

The stress time series are recordings of the total stress of single crystal $\mathrm{Cu}-10 \% \mathrm{Al}$ under compression at a constant strain rate (these time series were used in $/ 15 /$ ). The notation and some specifications for the data sets are given in Table 1. The selected records from the experiments regard plastic deformation giving successive slow up-load and rapid down-load of stress. 
Table 1

Notation and specification of the stress time series. The second column shows the temperature (T), the third column the strain rate $(\dot{\varepsilon})$, the fourth column the sampling time $\left(\tau_{s}\right)$ and the last column shows the recording time $(T)$.

\begin{tabular}{|c|c|c|c|c|}
\hline Notation & $\mathrm{T}\left[{ }^{\circ} \mathrm{C}\right]$ & $\dot{\varepsilon}\left[10^{-6} \mathrm{~s}^{-1}\right]$ & $\tau_{s}[\mathrm{~s}]$ & $T] \mathrm{s}]$ \\
\hline S1 & & 3.3 & 0.15 & 1000 \\
S2 & 300 & 37 & 0.006 & 30.28 \\
S3 & 300 & 107 & 0.06 & 400.02 \\
\hline
\end{tabular}

\section{REVIEW OF THE NONLINEAR ANALYSIS OF THE STRESS DATA}

Recently, it has been shown that the stress time series at low and medium strain rates are nonlinear and chaotic using standard nonlinear methods based on chaos theory $/ 8,5 /$. We start by reviewing these results on a particular time series, S1, described in Section 2.
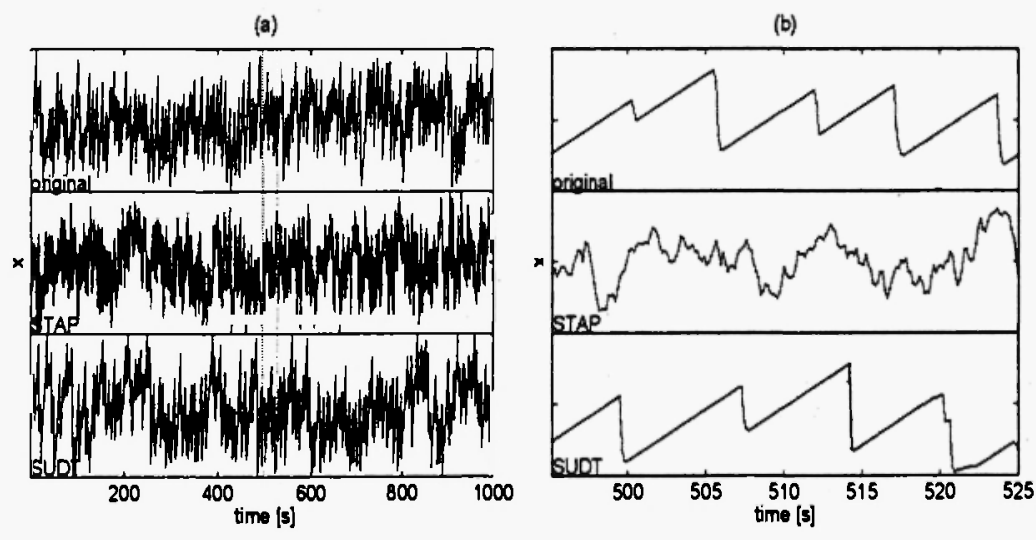

Fig. 1: (a) The stress time series, a STAP surrogate and a SUDT surrogate, from top to bottom. The two gray vertical lines denote the segments of the data, which are enlarged in (b).

The stress time series and a segment of this is shown in Fig. 1 (top panel). The structure of successive stick-slip patterns for this stress range clearly indicates that the underlying system is deterministic at small time scales. The other stress time series listed in Table 1 show the same feature of stick-slips. There is no ambiguity at the level of sampling time as to the evolution of the up-load stress (stick phase); it is simply a linear upward trend. The same holds for the slip phase, which is much shorter and thus the slope of the downward trend is very large. This fine piecewise linear stress evolution cannot be generated by conventional stochastic Markov chain models, such as ARMA models, neither by a linear deterministic system. It is 
therefore no surprise that the estimates from nonlinear methods applied to this type of time series suggest nonlinear deterministic structure (for a review on nonlinear methods refer to $/ 16,10,11$.

The presence of nonlinear short-term dynamics can also be established statistically, testing the null hypothesis $\mathrm{H}_{0}$ that the stress time series is generated by a linear (Gaussian) process, perturbed by a static, possibly nonlinear, transform $/ 12,13,14$. The transform is included in $\mathrm{H}_{0}$ to explain deviations from Gaussian amplitude distribution of the data, which is often observed in real time series. The test involves the generation of an ensemble of surrogate data, i.e. time series that represent the null hypothesis, and the computation of a test statistic $q$, here an estimate from a nonlinear method, on the original and surrogate data. If the estimate $q_{0}$ on the original data does not lie within the empirical distribution of $q$ under $\mathrm{H}_{0}$, formed by the estimates $q_{1}, q_{2}, \ldots, q_{M}$ on the $M$ surrogates, then $\mathrm{H}_{0}$ is rejected and it is unlikely that the original time series is linear stochastic. The statistics $q_{1}, q_{2}, \ldots, q_{M}$ form typically a normal-like distribution. Therefore the deviation of the statistic $q_{0}$ on the original data from the distribution of $q$ under $\mathrm{H}_{0}$ is quantified by the significance $S$ defined as

$$
S=\frac{\left|q_{0}-\bar{q}\right|}{s_{q}}
$$

where $\bar{q}$ and $s_{q}$ are the average and standard deviation of $q_{1}, q_{2}, \ldots, \tilde{z}_{M}$, respectively. The rejection region for $\mathrm{H}_{0}$ is formed by a lower limit for $S$ given from the critical value of standard normal distribution at a prespecified confidence level. If $S>1.96, \mathrm{H}_{0}$ is rejected at the $95 \%$ confidence level.

One could easily discriminate the surrogate data (consistent with the abovementioned $\mathrm{H}_{0}$ ) from the original stress data solely by eyeball judgement. As shown in Fig. 1 (middle panel), the surrogate time series fails to capture the special feature of the original data. The surrogate time series used in this Section are generated using the STAP algorithm, recently presented in $/ 17 /$. The same results were obtained using the AAFT and IAAFT algorithms (for a review on the algorithms and their performance see /18/. The surrogate data are designed to mimic the original time series in terms of amplitude distribution and autocorrelation and are otherwise random. These two conditions are apparently not sufficient to preserve the stick-slip patterns of the stress data.

In Fig. 2, we show estimates of the autocorrelation, mutual information and largest Lyapunov exponent on the original stress data and 40 STAP surrogate time series (for review on these methods see $/ 16,10,11$. The results on autocorrelation $r(\tau)$ for $\tau=1, \ldots, 50 \tau_{x}$ confirm that the STAP surrogate data have the same linear structure as the original time series. The other two measures are both nonlinear. The mutual information $I(\tau)$ measures the general correlation, linear and nonlinear. The discrepancy in $I(\tau)$ for the original and the surrogate data, shown in Fig. 2b, suggests that the original data contain nonlinear correlations and therefore give larger mutual information for a long range of lags. The largest Lyapunov exponent $\lambda_{1}(m)$ measures the

\footnotetext{
'The algorithms in the TISEAN software were used, see /19/.
} 
rate of divergence in the evolution of nearby trajectories in a reconstructed state space of dimension $m$. Chaotic and stochastic systems have positive $\lambda_{1}$, and large positive $\lambda_{1}$ indicate high complexity or stochasticity. As shown in Fig. 2c, the original data obtain significantly larger $\lambda_{1}(m)$ for the whole range of embedding dimensions $m=1, \ldots, 10$, which indicates that they exhibit more complexity than the surrogate data. - For both nonlinear statistics the significance $S$ takes very high values giving rejection of $\mathrm{H}_{0}$ at essentially $100 \%$ confidence level. The same results were established with other generation algorithms for the surrogate data and other nonlinear estimates, i.e. correlation dimension and fitting error of local averages.
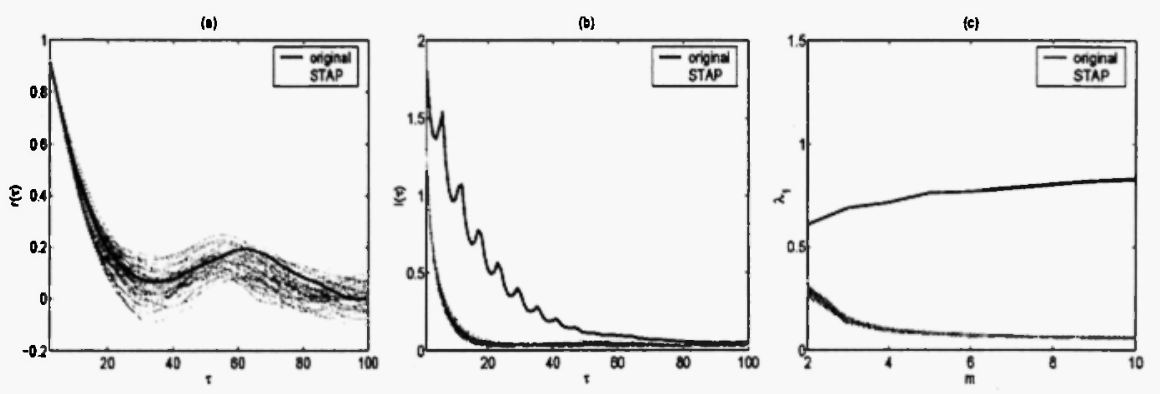

Fig. 2: Estimates on the stress time series and 40 STAP surrogates: in (a) autocorrelation $r(\tau)$ vs lag $\tau$, in (b) mutual information $/(\tau)$ vs $\tau$ and in (c) largest Lyapunov exponent $\lambda_{1}$ vs embedding dimension $m$.

The nonlinear time series analysis we have done so far could reproduce a quite evident result, i.e. the stress time series contains nonlinear dynamics at small time scale. A more interesting question we investigate next is whether there is any evidence of determinism or correlation in the evolution of the stick-slip patterns of the stress time series.

\section{SURROGATE DATA TEST FOR SEQUENCE OF PATTERNS}

We employ the statistical approach of surrogate data testing discussed in Section 3, but the working $\mathrm{H}_{0}$ now is that the succession of the stick-slip patterns is random, i.e. the stick-slip states are independent. The surrogate data for this $\mathrm{H}_{0}$ should have the same stick-slip structure as the stress data, but at a random order.

\subsection{The SUDT algorithm}

We have built an algorithm, called Stochastic Up-Down Trends (SUDT), to generate the surrogate data for this $\mathrm{H}_{0}$. The algorithm permutates randomly the stick-slips of the original time series taking care that the range of the original data is retained. Specifically, the steps of the algorithm are as follows: 
1. Scan the original time series $\mathbf{x}=\left[x_{1}, x_{2}, \ldots, x_{n}\right]^{\prime}$, identify and store the updown trend patterns, as well as the global minimum $x_{\min }$, the global maximum $x_{\max }$, the smallest end-point of the up-trend $x_{u}$ and the largest end point of the down-trend $x_{d}$.

2. The surrogate time series $\mathbf{z}$ to be constructed starts at the same data point as $\mathbf{x}$, i.e. $z_{1}=x_{1}$ (a random point could be chosen as well).

3. Using discrete uniform distribution, draw randomly an up-down trend segment from those stored in step 1 , and displace it so that its starting point coincides with the currently last point of the time series $\mathbf{z}$ (for the first iteration this is $z_{1}$ ).

4. Check whether the "up". end-point of the chosen up-down trend segment is between $x_{u}$ and $x_{\max }$, and the "down" end-point is between $x \min$ and $x_{d}$. If the two end-points are within the given limits, then accept the up-down trend (the "down" end-point of the accepted trend is now the last data point of the time series). If not, then discard the up-down trend and repeat step 3.

5. Repeat the last two steps until the time series $\mathbf{z}$ is as long as the original time series (eventually truncating the last eligible trend).

Note that the algorithm assumes that the original time series starts with an upward trend. We adjust accordingly the data sets by dropping a few samples from the beginning if necessary. Alternatively, one can simply reverse the magnitudes of the original data (e.g. multiply by -1) before applying the algorithm.

This algorithm implements bootstrapping on blocks of data, i.e. the up-down trends from the original time series, allowing repetitions of the same block of data in the surrogate time series. Simple shuffling of the original trends cannot be done because the end-points have to match.

It was found necessary to constrain the random selection of the up-down trends using lower and upper limits for both the "up" and "down" end-points of each trend in order to keep the generated surrogate time series $\mathbf{z}$ within the bounds of the original data. The use of the global minimum and maximum ( $x_{\min }$ and $\left.x_{\max }\right)$ alone led to edge effect problems, so the additional limits of $x_{u}$ and $x_{d}$ had to be introduced to assure robust execution of the algorithm. This increases the frequency of discarding randomly selected up-down trends and mars the random order of the up-down trends.

\subsection{Examples with simulated data}

The surrogate data generated by the SUDT algorithm represent the $\mathrm{H}_{0}$ of independent stick-slip states in the time series. Certainly, the stick-slip states of the original data may be correlated implying that the underlying mechanism exhibits a deterministic structure on longer time scales, which is an interesting possible aspect for the PLC effect.

Using simulated data, we show that the standard methods of time series analysis have actually discriminative power and can distinguish the original time series from its SUDT surrogates only when this is the case. For this, we use deterministic and stochastic time series of the stick-slip type. For the deterministic 
case, we use 2000 data of the log-transformed $w$ variable of the Rössler hyperchaos system $/ 20$, sampled at time $\tau_{s}=0.1 \mathrm{sec}$, call it $\mathbf{x}^{d}$. This time series exhibits stick-slip structure. For the stochastic case (time series with random stick-slip states), we simply use a time series derived by the SUDT algorithm on $\mathbf{x}^{\prime}$. call it $\mathbf{x}^{\mathrm{s}}$. Two segments of the two time series are shown in Fig. 3a. Note that the time series $x^{d}$ and $x^{5}$ have the same structure and cannot be distinguished by eyeball judgement. In the generation of 40 SUDT surrogates, there were on average 48 rejections of candidate stick-slips for $x^{d}$ (which is comprised of 193 stick-slip patterns) and about the same for $\mathbf{x}^{s}$, so that the shuffling can indeed be considered random. The SUDT surrogate data for each of the two time series possess similar amplitude distribution to the original ones, as shown in Fig. 3b. The same holds for the distribution of the up and down velocities and for the distribution of the up and down times. The preservation of all these distributions signifies the successful performance of the SUDT algorithm.

(a)

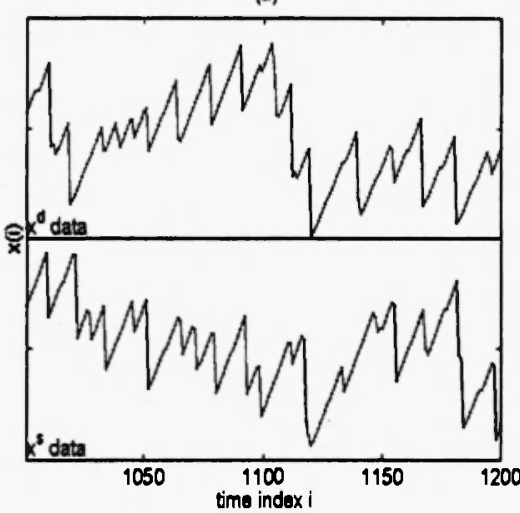

(b)

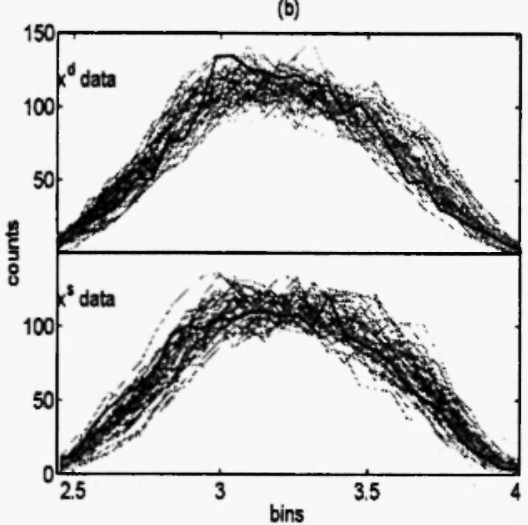

Fig. 3: (a) A segment of the time series of the Rössler hyperchaos system $\mathbf{x}^{d}$ (upper panel) and a segment of a SUDT surrogate of it $\mathbf{x}^{s}$ (bottom panel). (b) The amplitude distribution (histogram) of $x^{d}$ and 40 SUDT surrogates (upper panel), and of $x^{x}$ and 40 SUDT surrogates (lower panel). Black thick lines denote the original data and gray lines denote the surrogates.

We apply the linear and nonlinear test statistics on $\mathbf{x}^{d}, \mathbf{x}^{s}$ and their respective SUDT surrogates. The results are shown in Fig. 4. The autocorrelation $r(\tau)$ does not discriminate $x^{\gamma}$ from its SUDT surrogates, as expected, but the same holds for $\mathbf{x}^{d}$ (see Fig. 4a). Specifically for $\mathbf{x}^{d}$, rejection of $\mathrm{H}_{0}$ at the $95 \%$ confidence level could only be established for a small range of delays around $\tau=5$, as shown in Fig. $4 \mathrm{~d}$. So, for the chaotic time series from Rössler hyperchaos the linear test statistic has essentially no discriminative power. Note that there is no reason to believe that this is always the case with deterministic systems. However, the same results were obtained also on a quasi-periodic system with stick-slip structure (a two torus, for description of the system see $/ 21 \%$. 

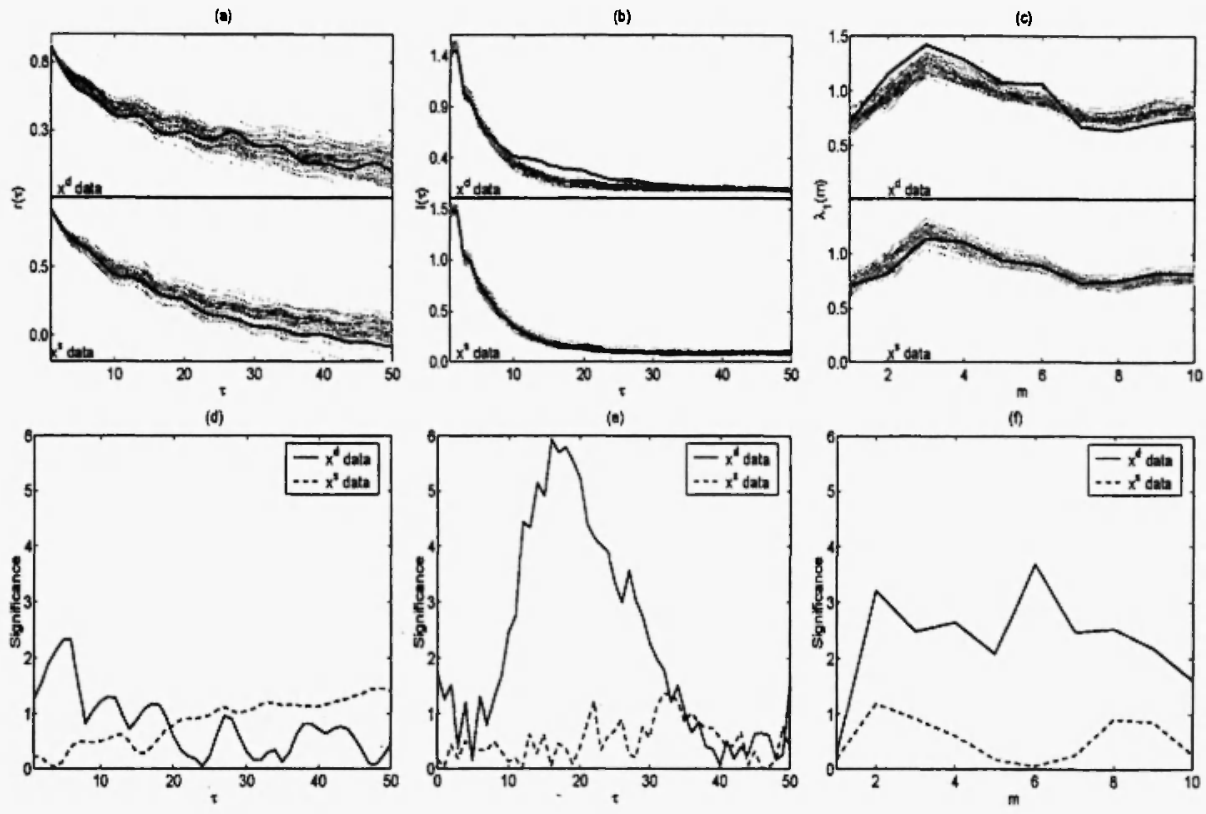

Fig. 4: (a) The autocorrelation $r(\tau)$ of $\mathbf{x}^{d}$ and 40 SUDT surrogates (upper panel), and of $x^{s}$ and 40 SUDT surrogates (lower panel). (b) The mutual information $I(\tau)$ for the same sets of data as for (a). (c) The largest Lyapunov exponent $\lambda_{1}(m)$ for the same sets of data as for (a). (d) The significance $S$ for $r(\tau)$ in (a). (e) $S$ for $l(\tau)$ in (b). (f) $S$ for $\lambda_{1}(m)$ in (c). For (a), (b) and (c), the black thick lines denote the original data and the gray lines denote the surrogates. For (d), (e) and (f), the level of $S=$ 1.96 is denoted by a horizontal gray line.

The chaotic deterministic data $\mathbf{x}^{d}$ are correctly distinguished from their respective SUDT surrogates with both nonlinear statistics, i.e. the mutual information $I(\tau)$ and the largest Lyapunov exponent $\lambda_{1}(m)$, as shown in Fig. $4 \mathrm{~b}$ and c. Subsequently, the $\mathrm{H}_{0}$ of independent stick-slip states is rejected at the $95 \%$ confidence levels for a long range of the free parameter of its statistic (see Fig. 4e and f). On the other hand, the $\mathbf{x}^{s}$ data are correctly not distinguished from their respective SUDT surrogates by either $\lambda_{1}(m)$ or $I(\tau)$, and $\mathrm{H}_{0}$ is not rejected for any value of the free parameter of the two statistics. Very similar results were obtained on the quasi-periodic system (where $S$ obtained larger values for the deterministic system) as well as when using other nonlinear statistics, e.g. local average maps and entropy.

These findings show that even standard nonlinear statistics that are not tailored for this particular test can distinguish correctly correlated stick-slip states from non-correlated stick-slip states of similar shape. 


\section{APPLICATION OF THE TEST TO THE STRESS DATA}

In order to avoid false local minima and maxima the stress time series were smoothed using a finite impulse response filter prior to the identification of the stick-slips. Note that there was no further use of the smoothed time series and the shuffling in the SUDT algorithm was done on the original stick-slips. The SUDT surrogate time series preserve well the stick-slip patterns of all the PLC stress time series. In Fig. 1, this is shown for the stress time series SI and one SUDT surrogate of this (upper and lower panel, respectively).

For the surrogate data to be proper for the test, we require good match of the distribution of the data, the distribution of the velocity of the up and down trends, as well as the distribution of the time of the up and down trends. The velocity and time distributions were preserved in the SUDT surrogates for all three stress time series. The amplitude distribution was well preserved for S2, sufficiently preserved for SI and not preserved for S3, as shown in Fig. 5. It turns out that the stick-slip time series generated by the SUDT algorithm tend to possess symmetric amplitude distribution, so that when the original data have skewed distribution (as is the case with S3) deviations in amplitude distribution do occur (see Fig. 5c). This constitutes a shortcoming of the SUDT algorithm to provide proper stick- slip surrogates. So, whenever the amplitude distribution is not preserved one may question the outcome of the test as deviations in the test statistics that may lead to rejection of $\mathrm{H}_{0}$ may be assigned to the mismatch of amplitude distribution.
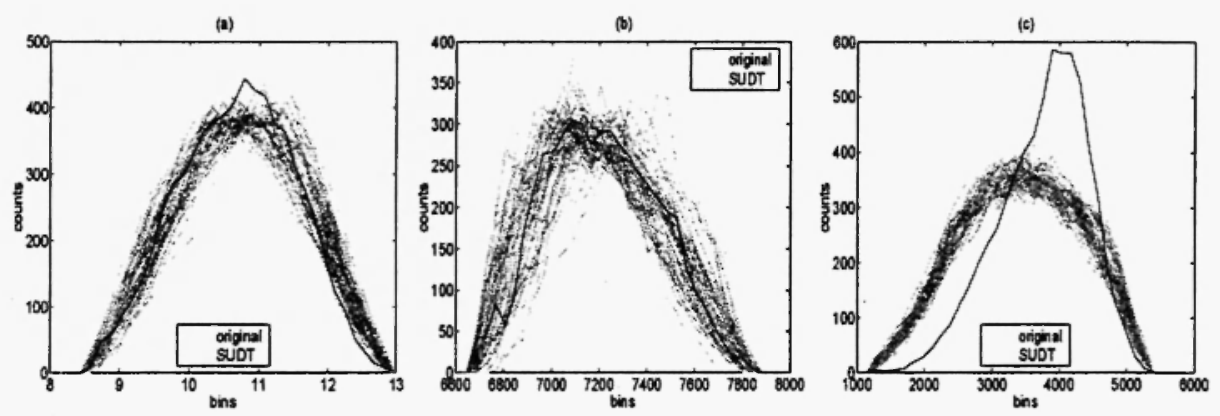

Fig. 5: Amplitude distribution of the three stress time series and their respective 40 SUDT surrogates: (a) S1, (b) S2, (c) S3.

In Fig. 6, the outcome of the test using the three test statistics is shown. The linear statistic $r(\tau)$ distinguishes the time series S1 from its 40 surrogates (for a long range of $\tau$ ), but not S2. For S3, the $r(\tau)$ for the SUDT surrogates is much higher (accordingly, $S$ takes very high values not shown in Fig. $6 \mathrm{~d}$ for $\tau \leq$ 13), but this mismatch may be due to the lack of match in amplitude distribution, so it cannot be regarded as genuine discrimination that would correspond to rejection of $\mathrm{H}_{0}$. Therefore the clear rejection of S3 also with the nonlinear statistics is not reliable. 

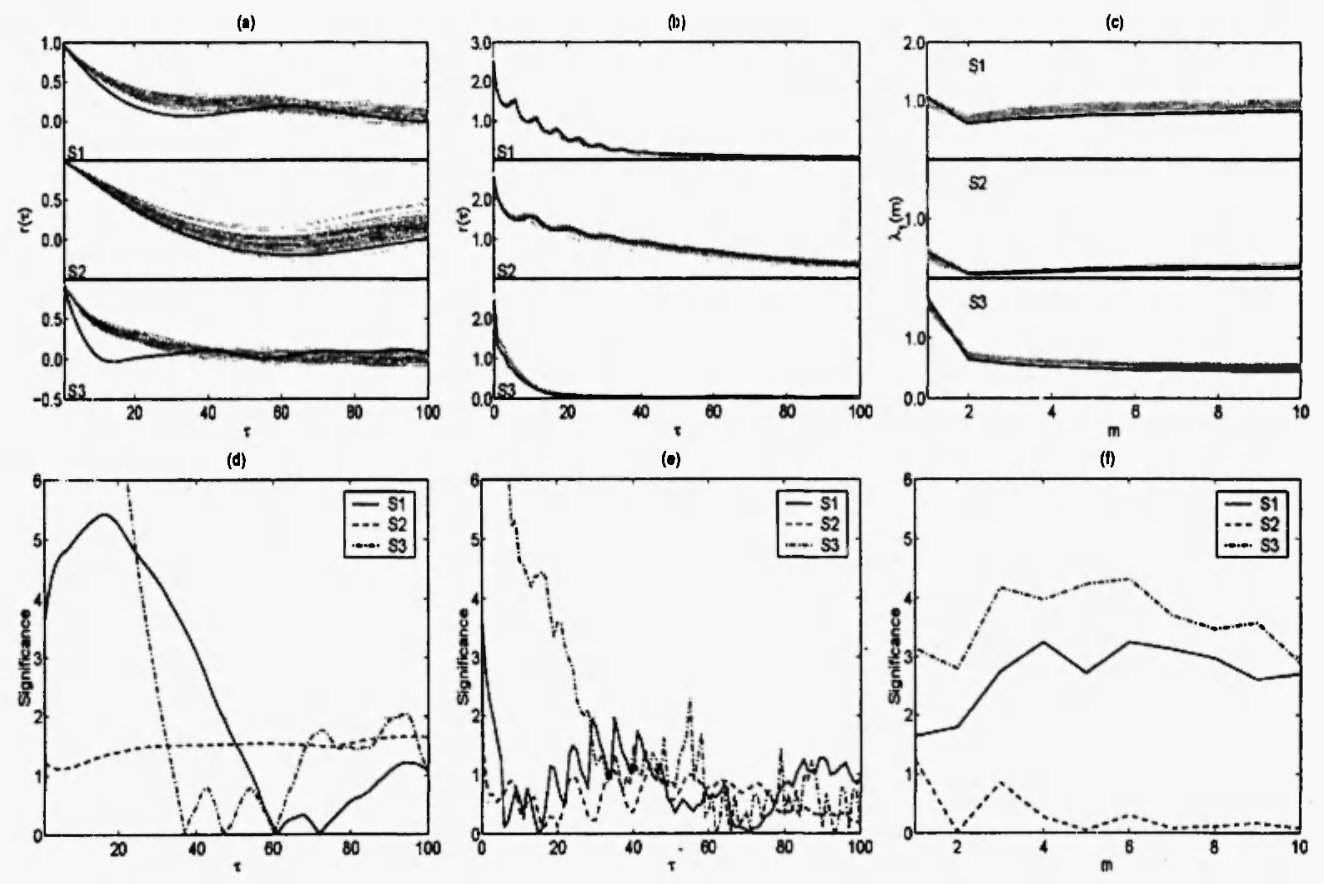

Fig. 6: (a) The autocorrelation $r(\tau)$ of the stress time series S1, S2, S3, and their 40 SUDT surrogates at the upper, middle and lower panel, respectively. (b) The mutual information $I(\tau)$ for the same sets of data as for (a). (c) The largest Lyapunov exponent $\lambda_{1}(m)$ for the same sets of data as for (a). (d) The significance $S$ for $r(\tau)$ in (a). (e) $S$ for $I(\tau)$ in (b). (f) $S$ for $\lambda_{1}(m)$ in (c). For (a), (b) and (c), the black thick lines denote the original data and the gray lines denote the surrogates. For (d), (e) and (f), the level of $S=1.96$ is denoted by a horizontal gray line.

It should be noted that for S1, the original $r(\tau)$ for $\tau<40$ is actually smaller in amplitude than for the surrogates suggesting the opposite of the alternative hypothesis we attempt to establish, i.e. the surrogate data involve more correlations than the original data. A possible explanation for this would be the discrepancy at the bulk of the amplitude distribution between S1 and its surrogates (see Fig. 5a). We note also that such long range correlations are often due to drift in the data.

The $I(\tau)$ statistic that measures both linear and nonlinear correlations is at the same level for $\mathrm{S} 1$ and its SUDT surrogates, as shown in Fig. 6b. Compared to the results with $r(\tau)$, it seems that S1 contains nonlinear correlations not present in the SUDT surrogates. However, $S$ is below the threshold for rejection of $\mathrm{H}_{0}$ at the $95 \%$ confidence level for all but very small $\tau$ (see Fig. 6e). The $\lambda_{1}(m)$ statistic shows also a difference in nonlinear correlations between $\mathrm{S} 1$ and its surrogates giving confident rejections at the $95 \%$ level for $m>2$, as shown in Fig. $6 \mathrm{c}$ and $\mathrm{f}$.

The stress time series S2 could not be discriminated from its SUDT surrogates with any of the three statistics, indicating that it has no correlations between stick-slips. 
The results on the three stress time series suggest that there is not enough statistical evidence to establish that the stick-slip states of the stress time series from plastic deformation of single crystals are correlated and thus that there is a deterministic system at large time scales that controls the evolution of the stick-slip states.

\section{DISCUSSION}

It is plausible that the evolution of the stress in the PLC effect is rather deterministic at short time scales as the stress time series has a characteristic stick-slip structure. We employed statistical testing to investigate whether there is a deterministic mechanism that controls the stress at larger time scales that span over the duration of the stick-slip states. The standard surrogate data test for nonlinearity, used recently to establish determinism and nonlinearity for the PLC effect, is not suitable for the question of interest as the surrogate data do not preserve the stick-slip structure. Subsequently, the discrimination between original stress time series and surrogate data (generated under the null hypothesis of linear stochastic system) is guaranteed. However, this result does not establish the presence of correlations between the stick-slips that should be present if the underlying mechanism is nonlinear deterministic. Indeed, our simulations showed that time series comprised of uncorrelated stick-slips are also discriminated from this type of surrogates, questioning the appropriateness of the surrogate data test for nonlinearity for this type of time series.

We designed the SUDT algorithm to generate surrogate data of stick-slip structure and performed the surrogate data test for the null hypothesis of independent stick-slip states. Nonlinear statistics such as the ones used for the test for nonlinearity turned out to perform appropriately when applied to simulated data. However, the power of the statistics on chaotic time series with stick-slip structure was not as high as for the quasi-periodic systems.

We applied this test on three stress time series from plastic deformation of single crystal $\mathrm{Cu}-10 \% \mathrm{Al}$ under compression at different strain rates. We used one linear test statistic, i.e. the autocorrelation, and two nonlinear statistics: the mutual information and the largest Lyapunov exponent. The null hypothesis could be rejected, but not clearly, for the stress time series obtained at low constant strain rate $\left(\dot{\varepsilon}=3.3 \cdot 10^{-6} \mathrm{~s}^{-1}\right)$ and could not be rejected with any test statistic for the stress time series obtained at medium constant strain rate $\left(\dot{\varepsilon}=37 \cdot 10^{-6} \mathrm{~s}^{-1}\right)$. For the third time series at larger constant strain rate $\left(\dot{\varepsilon}=107 \cdot 10^{-6} \mathrm{~s}^{-1}\right)$, the SUDT algorithm failed to match the amplitude distribution and thus the rejection obtained with the test statistics is questioned. Overall, the statistical testing could not establish that the stress time series contain significant correlations. However, this is a pilot study and a systematic application of the test to stress time series under varying experimental conditions is planned.

An improvement of the SUDT algorithm would be to constrain the surrogates to match the amplitude distribution of the original time series, but there does not seem to be an obvious way to do this. Our simulations showed that the match is maintained through the shuffling of the stick-slips, but for one stress time series this failed. Also, the test may be improved by employing other test statistics that are tailored to 
capture the information relating the stick-slip states, such as correlation between the lengths of successive updown trends or between the magnitudes of successive turning points.

\section{ACKNOWLEDGMENTS}

Partial support of the European Community's Human Potential Programme under contract HPRN-CT2002-00198 (DEFINO) and research interaction between AUTh/Greece and TUB/Germany are acknowledged.

\section{REFERENCES}

1. G. F. Bell. The experimental foundations of solid mechanics. In S. Flügge and C. Truesdall, editors, Encyclopedia of Physics, volume Vla/l of Mechanics of Solids I. Springer-Verlag, Berlin, 1973.

2. E. C. Aifantis. The Physics of plastic deformation. Int. J. Plast., 3:211-247, 1987.

3. H. M. Zbib and E. C. Aifantis. A gradient-dependent model for the Portevin-Le Châtelier effect. Scripta Met., 22:1331 - 1336, 1988.

4. M. S. Bharathi, M. Lebyodkin, G. Ananthakrishna, C. Fressengeas, and L. P. Kubin. The hidden order behind jerky flow. Acta Materialia, 50:2813 - 2824, 2002.

5. M. Lebyodkin, L. Dunin-Barkowskii, Y. Brechet, Y. Estrin, and L. P. Kubin. Spatio-temporal dynamics of the Portevin-Le Châtelier effect: Experiment and modelling. Acta Materialia, 48:2529 $-2541,2000$.

6. P. Hähner, A. Ziegenbein, E. Rizzi, and H. Neuhäuser. Spatiotemporal analysis of Portevin-Le Châtelier deformation bands: Theory, simulation, and experiment. Physical Review B, 65, 134109 (2002).

7. G. Ananthakrishna, C. Fresengeas, and L. P. Kubin. Chaos and the jerky flow in Al-Mg polycrystals. Materials Science and Engineering, A234-236:314 - 337, 1997.

8. G. Ananthakrishna, S. J. Noronha, C. Fresengeas, and L. P. Kubin. Crossover from chaotic to selforganized critical dynamics in jerky flow of single crystals. Physical Review E, 60(5):5455 - 5462, 1999.

9. S. J. Noronha, G. Ananthakrishna, L. Quaouire, C. Fressengeas, and L. P. Kubin. Chaos in the Portevin-Le Châtelier effect. International Journal of Bifurcation and Chaos, 7:2577-2586, 1997.

10. H. D. I. Abarbanel. Analysis of Observed Chaotic Data. Springer, New York, 1996.

11. H. Kantz and T. Schreiber. Nonlinear Time Series Analysis. Cambridge University Press, Cambridge, 1997.

12. J. Theiler, S. Eubank, A. Longtin, and B. Galdrikian. Testing for nonlinearity in time series: the 
method of surrogate data. Physica D, 58:77 - 94, 1992.

13. T. Schreiber and A. Schmitz. Surrogate time series. Physica D, 142(3-4):346 - 382, 2000.

14. D. Kugiumtzis. Surrogate data test on time series. In A. Soofi and L. Cao, editors, Modelling and Forecasting Financial Data, Techniques of Nonlinear Dynamics, chapter 12, pages $267-282$. Kluwer Academic Publishers, 2002.

15. G. Ananthakrishna, C. Fresengeas, M. Grosbras, J. Vergnol, C. Engelke, J. Plessing, H. Neuhauser, E. Bouchard, J. Planes, and L.P. Kubin. On the existence of chaos in jerky flow. Scripta Metallurgica et Materialia, 32(11):1731 - 1737, 1995.

16. D. Kugiumtzis, B. Lillekjendlie, and N. Christophersen. Chaotic time series part I: Estimation of some invariant properties in state space. Modeling, Identification and Control, 15(4): $205-224$, 1994.

17. D. Kugiumtzis. Statically transformed autoregressive process and surrogate data test for nonlinearity. Physical Review E, 66:025201, 2002.

18. D. Kugiumtzis. On the reliability of the surrogate data test for nonlinearity in the analysis of noisy time series. International Journal of Bifurcation and Chaos, 11(7):1881 - 1896, 2001.

19. R. Hegger, H. Kantz, and T. Schreiber. Practical implementation of nonlinear time series methods: The TISEAN package. Chaos, 9:413, 1999.

20. O. E. Rössler. An equation for hyperchaos. Physics Letters A, 71(2 - 3):155 - 157, 1979.

21. A. M. Fraser. Reconstructing attractors from scalar time series: a comparison of singular system and redundancy criteria. Physica D, 34:391 - 404, 1989. 
